\title{
Experimental Implementation of Remote State Preparation by Nuclear Magnetic Resonance
}

\author{
Xinhua Peng ${ }^{1}$, Xiwen $_{Z^{1}}{ }^{*}$, Ximing Fang ${ }^{2,1}$, Mang Feng ${ }^{1}$, Maili Liu ${ }^{1}$, and Kelin Gao ${ }^{1}$ \\ ${ }^{1}$ Laboratory of Magnetic Resonance and Molecular Physics, \\ Wuhan Institute of Physics and Mathematics, the Chinese Academy of Sciences, \\ Wuhan, 430071, People's Republic of China \\ ${ }^{2}$ Department of Physics, Hunan Normal University, \\ Changsha, 410081, People's Republic of China
}

\begin{abstract}
We have experimentally implemented remote state preparation (RSP) of a qubit from a hydrogen to a carbon nucleus in molecules of carbon-13 labeled chloroform ${ }^{13} \mathrm{CHCl}_{3}$ over interatomic distances using liquid-state nuclear magnetic resonance (NMR) technique. Full RSP of a special ensemble of qubits, i.e., a qubit chosen from equatorial and polar great circles on a Bloch sphere with Pati's scheme, was achieved with one cbit communication. Such a RSP scheme can be generalized to prepare a large number of qubit states and may be used in other quantum information processing and quantum computing.
\end{abstract}

Typeset using REVTEX

*Corresponding author. E-mail:xwzhu@wipm.whcnc.ac.cn; Fax: 0086-27-87885291. 


\section{INTRODUCTION}

Quantum states are the carrier of quantum information. Preparing, controlling, accessing and transmitting quantum states constitute the important part of quantum information theory. However, the non-clone theorem [1] of an unknown quantum state holds back the effective utilization of quantum information. One of the striking discoveries in this area is quantum teleportation [2], which transmits an unknown quantum state from the sender (Alice) to the receiver (Bob) by a maximally entangled channel (ebit) and two classical bit (cbit) communication, namely 1 ebit+2 cbit teleports 1 qubit. Recently, some interesting works for remote state preparation (RSP) [7] 9], similar to teleportation, have been theoretically proposed by Lo [8], Pati [7] and Bennett et al. [9], which can remotely prepare a quantum state from Alice to Bob with less classical bit communication than teleportation. In their papers, they showed that for a special ensemble of states (e.g., qubit states chosen from the equator or the polar of the Bloch sphere) RSP requires only one bit of classical communication per qubit, exactly half that of teleportation, i.e., 1 ebit +1 cbit transmits 1 qubit. But hitherto, though quantum teleportation has been implemented experimentally [3], RSP has not been experimentally tested yet.

In this paper, we will experimentally demonstrate RSP for a special quantum state (i.e., a qubit chosen from equator or polar great circles of a Bloch sphere), with a 2-qubit sample, carbon-13 labeled chloroform ${ }^{13} \mathrm{CHCl}_{3}$, and complete the task that 1 ebit +1 cbit transmits 1 qubit. For the general case, Lo [8] conjectured that the classical communication cost of RSP is equal to that of teleportation, and their procedure would be similar. Bennett et al. [9] showed that, in the presence of a large amount of prior entanglement, the asymptotic classical communication cost of RSP for general states is one bit per qubit. However, the experimental verification of the asymptotic character requires preparation of a large amount of entanglement source, which would be difficult to implement by the existing techniques. 


\section{REMOTE STATE PREPARATION OF A SPECIAL ENSEMBLE AND THE NMR REALIZATION}

Consider an arbitrary single qubit state

$$
|\psi\rangle=\alpha|0\rangle+\beta|1\rangle
$$

where $\alpha=\cos (\theta / 2)$ is chosen to be real and $\beta=\sin (\theta / 2) \exp (i \phi)$, a complex number. The state can be represented by a point in a Bloch sphere with two real parameters $\theta$ and $\phi$, and obtained by a transformation $R$ on $|0\rangle$ with

$$
R=\left(\begin{array}{cc}
\cos (\theta / 2) & -\sin (\theta / 2) e^{-i \phi} \\
\sin (\theta / 2) e^{i \phi} & \cos (\theta / 2)
\end{array}\right)
$$

The task of RSP is that Alice helps Bob in a distant laboratory to prepare the state $|\psi\rangle$ known to her but unknown to Bob.

Pati [7] proposed a procedure for RSP of a qubit chosen from equatorial or polar line on a Bloch sphere. The schematic circuit for RSP is shown in Fig. 1(a).

Similar to teleportation, at first, Alice and Bob share a two-qubit maximal entangled state, i.e. an EPR state,

$$
\left|\psi^{-}\right\rangle_{A B}=\frac{1}{\sqrt{2}}\left(|0\rangle_{A}|1\rangle_{B}-|1\rangle_{A}|0\rangle_{B}\right)
$$

$\left|\psi^{-}\right\rangle_{A B}$ can be expanded in different orthogonal basis. We choose the qubit orthogonal basis $\left\{|\psi\rangle,\left|\psi_{\perp}\right\rangle\right\}$

$$
\begin{aligned}
& \left|\psi^{-}\right\rangle_{A B}=\frac{1}{\sqrt{2}}\left(|\psi\rangle_{A}\left|\psi_{\perp}\right\rangle_{B}-\left|\psi_{\perp}\right\rangle_{A}|\psi\rangle_{B}\right) \\
& =\frac{1}{\sqrt{2}}\left(|\psi\rangle_{A} U^{-1}|\psi\rangle_{B}-\left|\psi_{\perp}\right\rangle_{A}|\psi\rangle_{B}\right) .
\end{aligned}
$$

where $\left|\psi_{\perp}\right\rangle=\alpha|1\rangle-\beta^{*}|0\rangle, \beta^{*}$ is the complex conjugate of $\beta$, and $U^{-1}$ is the inverse of $U$ which is a unitary operator to convert $\left|\psi_{\perp}\right\rangle$ into $|\psi\rangle$. It is possible for Alice to perform a single particle Von-Neumann measurement under the basis because of the state $|\psi\rangle$ known to her. Conditional on Alice's two measurement outcomes $|\psi\rangle$ and $\left|\psi_{\perp}\right\rangle$, Bob's state is 
either $|\psi\rangle$ or $U^{-1}|\psi\rangle$. Thus, after Alice performs the measurement, she sends the result (one classical bit information) to Bob, who can then recover the desired state $|\psi\rangle$ by deciding to do nothing $E$ or a unitary operation $U$ according to the received classical information. For a special ensemble, $U$ can be an affirmative unitary operator to transform $\left|\psi_{\perp}\right\rangle$ into $|\psi\rangle$. For example, if Alice choose to prepare a polar great circles on a Bloch sphere, i. e., $\phi=0,|\psi\rangle=\cos (\theta / 2)|0\rangle+\sin (\theta / 2)|1\rangle$, then $U=i \sigma_{y}$. Alternatively, if Alice wish to help Bob prepare an arbitrary equatorial state such as $|\psi\rangle=\frac{1}{\sqrt{2}}\left(|0\rangle+e^{i \phi}|1\rangle\right)$, then $U=\sigma_{z}$. However, for a general state (both $\theta$ and $\phi$ are arbitrary), there is no universal transformation $U$ to take $\left|\psi_{\perp}\right\rangle \rightarrow|\psi\rangle$ by this protocol. Therefore, RSP of an arbitrary state can be made with the success probability of $50 \%$.

The network of RSP for a special ensemble shown in Fig. 1(b) corresponds to the schematic circuit in Fig.1(a), including three steps.

(1) Preparation of the EPR pair $\left|\psi^{-}\right\rangle_{A B}$. In NMR experiments, suppose that Alice and Bob have spin $A$ and spin $B$, respectively, consisting of a bipartite spin quantum system in a $4 \times 4$ Hilbert space $H_{A} \otimes H_{B}$. The EPR pair can be prepared from a pure state $|0\rangle_{A}|0\rangle_{B}$ by the operations of NOT ( $N_{i}$ flips the sign of spin $i$ ), the Hadamard gate $\left(H_{i}\right.$ transform the states according to $\left(|0\rangle \rightarrow \frac{1}{\sqrt{2}}(|0\rangle+|1\rangle),|1\rangle \rightarrow \frac{1}{\sqrt{2}}(|0\rangle-|1\rangle)\right)$ and controlled-NOT $\left(C N_{\bar{A} B}\right.$ flips spin $B$ if and only if $A$ is $|0\rangle)$ gates. The $N_{A}, H_{A}$ and $C N_{\bar{A} B}$ gates were respectively realized by the pulse sequences $X_{A}(\pi), \bar{Y}_{A}\left(\frac{\pi}{2}\right) \bar{X}_{A}(\pi)$ and $\bar{Y}_{B}\left(\frac{\pi}{2}\right) Z_{B}\left(\frac{\pi}{2}\right) Z_{A}\left(\frac{\pi}{2}\right) J_{A B}(\pi)$ (pulses applied from right to left). By simplification methodology [12, the EPR pair was realized by using the pulse sequence

$$
\bar{Y}_{B}\left(\frac{\pi}{2}\right) J_{A B}(\pi) Y_{B}\left(\frac{\pi}{2}\right) \bar{X}_{B}\left(\frac{\pi}{2}\right) \bar{Y}_{A}(\pi) X_{A}\left(\frac{\pi}{2}\right)
$$

(2) The single particle Von-Neumann measurement under the qubit basis $\left\{|\psi\rangle,\left|\psi_{\perp}\right\rangle\right\}$. We use a two-part procedure inspired by Brassard et al. to perform the measurement [10]. Part one of the procedure is to rotate from the basis $\left\{|\psi\rangle,\left|\psi_{\perp}\right\rangle\right\}$ into the computational basis $\{|0\rangle,|1\rangle\}$ by the transformation $R^{+}=R^{-1}$, inverse of $R$. Part two of the procedure is to perform a projective measurement in the computational basis. 
Applying $R_{A}^{+}$on the EPR pair, one gets

$$
R_{A}^{+}\left|\psi^{-}\right\rangle_{A B} \rightarrow \frac{1}{\sqrt{2}}\left(|0\rangle_{A}\left|\psi_{\perp}\right\rangle_{B}-|1\rangle_{A}|\psi\rangle_{B}\right)
$$

For a polar state, the operation

$$
R_{A}^{+}(\theta, \phi=0)=\left(\begin{array}{cc}
\cos (\theta / 2) & \sin (\theta / 2) \\
-\sin (\theta / 2) & \cos (\theta / 2)
\end{array}\right)
$$

which can be realized by the NMR pulse sequence $\bar{Y}_{A}(\theta)$. For an equatorial state, the operation

$$
R_{A}^{+}\left(\theta=90^{\circ}, \phi\right)=\frac{1}{\sqrt{2}}\left(\begin{array}{cc}
1 & e^{-i \phi} \\
-e^{i \phi} & 1
\end{array}\right)
$$

which can be realized by the NMR pulse sequence $X_{A}\left(\theta_{1}\right) \bar{Y}_{A}\left(\theta_{2}\right) X_{A}\left(\theta_{1}\right)$, where $\theta_{1}=$ $\tan ^{-1}(\sin \phi)$, and $\theta_{2}=2 \sin ^{-1}(\cos \phi / \sqrt{2})$.

It is possible to simulate directly the second step in NMR exploiting magnetic gradient pulses [11]. However, it can be seen from Eq. (6) that, the final state of Alice is one of $|0\rangle$ and $|1\rangle$, corresponding to the two different measurement results. Owing to the weak measurement of NMR, we can employ the conditional unitary operation $S$, instead of the projective measurement in the computational basis and the post-measurement operation.

(3) Conditional unitary operation $S$. It can be verified from Eq. (6), that when Alice measures the outcome of spin $A|0\rangle$, Bob performs the transformation $U$ on spin $B$; when $|1\rangle$, Bob does nothing, then two measurement results lead to Bob in $|\psi\rangle$. For a polar state, $U=i \sigma_{y}$, the conditional unitary operation $S=i \sigma_{y}^{B} E_{+}^{A}+E_{-}^{A}$, is implemented by the pulse sequence

$$
\bar{Y}_{B}\left(\frac{\pi}{2}\right) X_{B}\left(\frac{\pi}{2}\right) J_{A B}(\pi) \bar{X}_{B}\left(\frac{\pi}{2}\right)
$$

For an equatorial state, $U=\sigma_{z}$, the conditional unitary operation $S=\sigma_{z}^{B} E_{+}^{A}+E_{-}^{A}$, is implemented by the pulse sequence

$$
\bar{Y}_{A}\left(\frac{\pi}{2}\right) \bar{X}_{A}\left(\frac{\pi}{2}\right) Y_{A}\left(\frac{\pi}{2}\right) \bar{Y}_{B}\left(\frac{\pi}{2}\right) X_{B}\left(\frac{\pi}{2}\right) Y_{B}\left(\frac{\pi}{2}\right) J_{A B}(\pi)
$$


(4) The measurement of the final state. After tracing out spin $A$ the state of spin $B$ is reduced to the expected form $|\psi\rangle$, which indicates the success of RSP. In principle, spin $A$ could be traced out experimentally by applying a decoupling field during observation of spin $B$. But this approach was found to lead to unacceptable sample heating, with resultant shifts in resonance frequencies. Thus, the tracing out process was implemented in software by integrating the entire multiplet of spin $\mathrm{B}$ after adjusting the right phase.

\section{THE NMR EXPERIMENT AND RESULTS}

The RSP scheme stated above was implemented by liquid-state NMR spectroscopy with carbon-13 labeled chloroform ${ }^{13} \mathrm{CHCl}_{3}$ (Cambridge Isotope Laboratories, Inc.). To perform RSP we make use of the hydrogen nucleus $\left({ }^{1} H\right)$ as the sender (Alice) and the carbon nuclei $\left({ }^{13} C\right)$ as the receiver (Bob) in the experiments, transmitting the state from ${ }^{1} H$ to ${ }^{13} C$. Spectra were recorded on a BrukerARX500 spectrometer with a probe tuned at $125.77 \mathrm{MHz}$ for ${ }^{13} \mathrm{C}$, and at $500.13 \mathrm{MHz}$ for the ${ }^{1} \mathrm{H}$. The spin-spin coupling constant $J$ between ${ }^{13} \mathrm{C}$ and ${ }^{1} \mathrm{H}$ is $214.95 \mathrm{~Hz}$. The relaxation times were measured to be $T_{1}=4.8 \mathrm{sec}$ and $T_{2}=0.2 \mathrm{sec}$ for the proton, and $T_{1}=17.2 \mathrm{sec}$ and $T_{2}=0.35 \mathrm{sec}$ for carbon nuclei.

The pseudo-pure state was prepared in our experiment using line-selective pulses and the gradient-pulse techniques [13]. We performed two separate sets of experiments of the RSP process (shown in Fig.2) from ${ }^{1} H$ to ${ }^{13} C$. As all NMR observables are traceless and the constant item has no effect on the NMR signal, any point on the Bloch sphere can be expressed as the product form $I_{x} \sin \theta \cos \phi+I_{y} \sin \theta \sin \phi+I_{z} \cos \theta$ (apart from a constant unit matrix), which corresponds to the observable NMR signal. Using Alice's knowledge of the qubit, an advanced operation $R^{+}$was performed on ${ }^{1} H$ for a given set of $\theta$ and $\phi$ values. In the first set, a total of 25 qubit states chosen from a range of points on the polar line of the Bloch sphere $(\phi=0)$ with a $\theta$ spacing of $\pi / 12$ were studied. The second set of experiments was executed for RSP of 17 equatorial states on the Bloch sphere $(\theta=\pi / 2)$ with a $\phi$ spacing of $\pi / 8$. For each state the experimental spectra of ${ }^{13} C$ were recorded from the ${ }^{13} C$ 
nucleus, and their real and imaginary components are plotted in Fig. 2. The experimental results clearly show the expected line, sine and cosine modulations. It can be seen from Fig. 2, there exists an same initial phase offset in the fitting curves, which maybe caused by the systemic errors, such as static magnetic field and rf field inhomogeneities and the imperfect calibrations of rf pulses, etc.. Furthermore, we reconstructed the density matrices of the obtained states by quantum state tomography [14], and the maximal relative errors $\triangle<18 \%$, where

$$
\triangle=\max _{\text {all states }}\left\{\frac{\left\|\rho_{\text {theory }}-\rho_{\exp t}\right\|}{\left\|\rho_{\text {theory }}\right\|}\right\},
$$

and $\rho_{\text {theory }}$ is the expectative state of RSP, and $\rho_{\exp t}$ is the reconstructed state by the experimental data. Besides static magnetic field and rf field inhomogeneities and the imperfect calibrations of rf pulses, the errors in experiments arise from decoherence due to the $T_{2}$ relaxations of nuclei and the environmental effects such as temperature. Nevertheless, it turns out from Fig. 2 and the reconstructed density matrices that the RSP network is effective for a special ensemble.

\section{CONCLUSION}

In summary, we have experimentally implemented the RSP protocol by using NMR quantum logic gates and circuits in quantum information. In our experiments, RSP of a special ensemble was successfully completed by a maximally entangled channel with one classical bit communication, exactly half that of teleportation, namely 1 cbit $\rightarrow 1$ qubit. The special states distributes on the equatorial and polar circles on the Bloch sphere. The RSP

protocol can be generalized to remotely prepare a large number of general qubit states in the high-entanglement limit at an asymptotic cost of one bit per qubit [9]. Like teleportation, RSP can be applied not only to pure states, but also to parts of entangled states which has been studied by Bennett et al. [9]. Although the communication happens between spins in angstrom distance, the concept and method of quantum information transmission should be useful for quantum computation and quantum communication. 


\section{ACKNOWLEDGMENTS}

We thank C. P. Sun for bringing the topic of RSP to our attention and Xiaodong Yang, Hanzheng Yuan, Xu Zhang and Guang Lu for help in the course of experiments. 


\section{REFERENCES}

[1] W. K. Wootters and W. H. Zurek, Nature 299, 802 (1982).

[2] C. H. Bennett, G. Brassard, C.Crepeau, R. Jozsa, A. Peres, and W. K. Wootters, Phys. Rev. Lett. 70, 1895 (1993).

[3] M. A. Nielsen, E. Knill and R. Laflamme, Nature 396, 52 (1998).

[4] D. Bouwmeester et al. Nature 390, 575 (1997).

[5] D. Boschi, S. Branca, F. De Martini, L. Hardy and S. Popescu, Phys. Rev. Lett. 80, 1121 (1998).

[6] X. Fang, X. Zhu, M. Feng, X. Mao and F. Du, Phys. Rev. A (2000).

[7] Arun K. Pati, Phys. Rev. A 63, 014302 (2000).

[8] Hoi-Kwong Lo, Phys. Rev. A 62, 012313 (2000).

[9] C. H. Bennett, D. P. DiVincenzo, J. A. Smolin, B. M. Terhal and W. K. Wootters, Phys. Rev. Lett. 87, 077902 (2001).

[10] G. Brassard, S. Braunstein and R. Cleve, Phys. D 120, 43 (1998).

[11] G. Teklemariam, E. M. Fortunato, M. A. Pravia, T. F. Havel and D. G. Cory, Phys. Rev. Lett. 86, 5845 (2001).

[12] R. Ernst, G. Bodenhausen and A. Wokaun, Principles of Nuclear Magnetic Resonance in One and Two Dimensions (Oxford Univ. Press, Oxford, 1990).

[13] X. Peng, X. Zhu, X. Fang, M. Feng, K. Gao, X. Yang and M. Liu, Chem. Phys. Lett. 340, 509 (2001).

[14] I. L. Chuang, N. Gershenfeld, M. Kubinec and D. Leung, Proc. Roy. Soc. Lond A 454 447 (1998).

[15] B. Schumacher, Phys. Rev. A 54, 2614 (1996). 


\section{Figure Captions}

Fig. 1 Schematic protocol for RSP proposed by Pati (a) and the corresponding network of implementing RSP (b). $H$ represents the Hadamard gate and conditional operation on a spin being in the $|1\rangle$ state and the $|0\rangle$ state are represented by a filled circle and an empty circle, respectively. $R^{+}$is demonstrated in the text.

Fig. 2 Experimental results for RSP of qubits chosen from (a) the polar line, and (b) the equatorial circle, on the Bloch sphere. Data points $\bigcirc$ and $*$ denote the real and imaginary parts of the NMR signals from ${ }^{13} C$, respectively. The fitting expectative curves are depicted with the solid lines. 


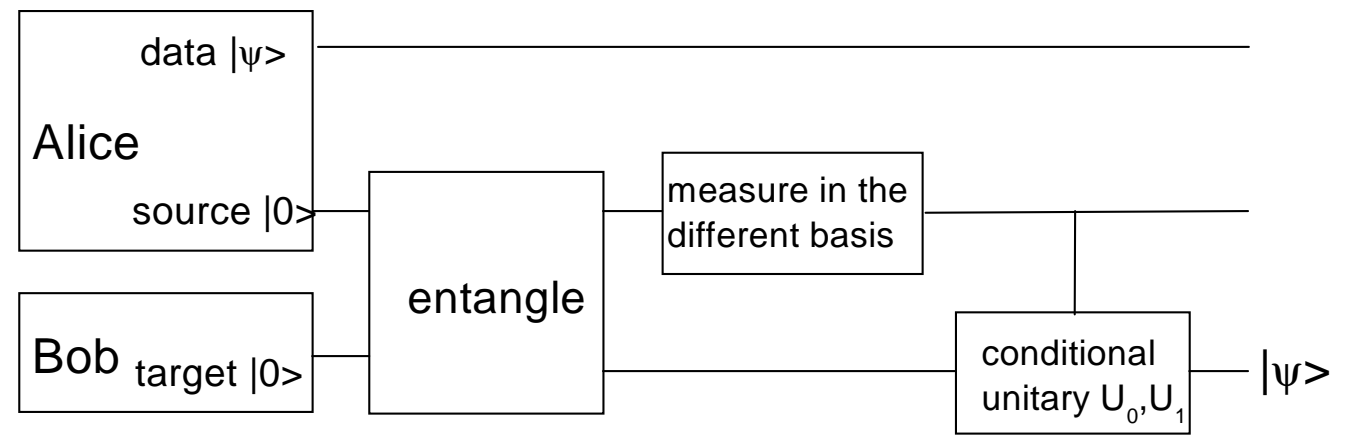

(a)

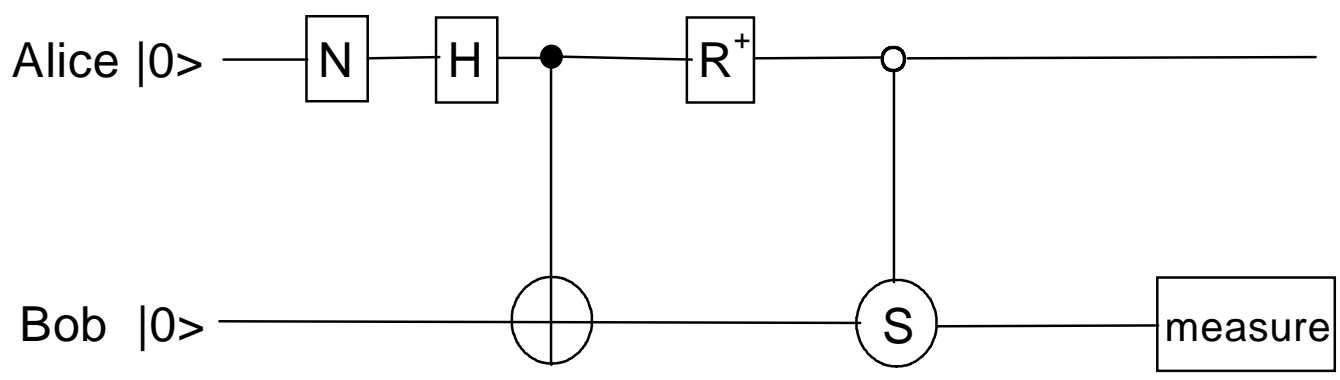

(b)

\section{Fig. 1}


(a)

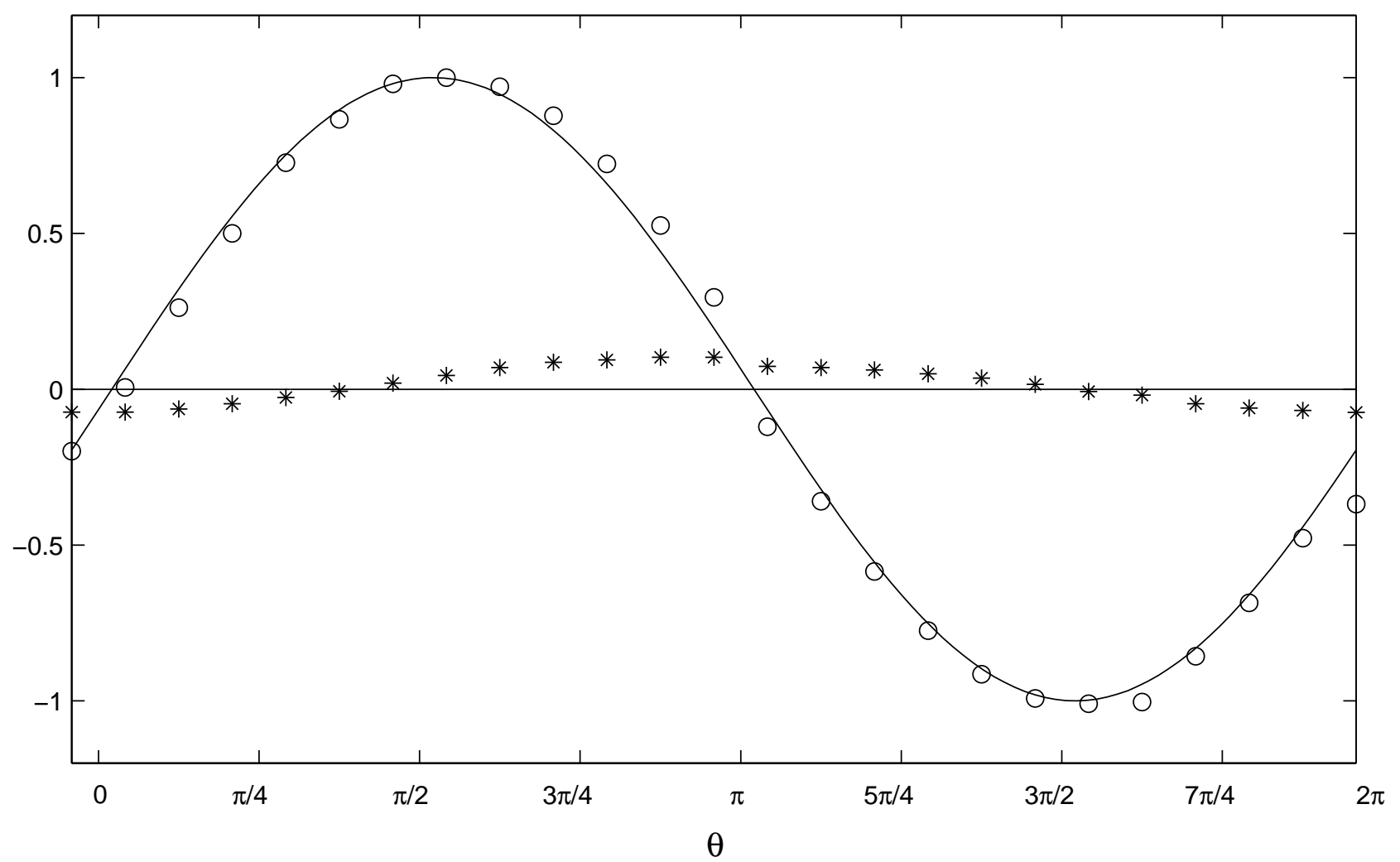

(b)

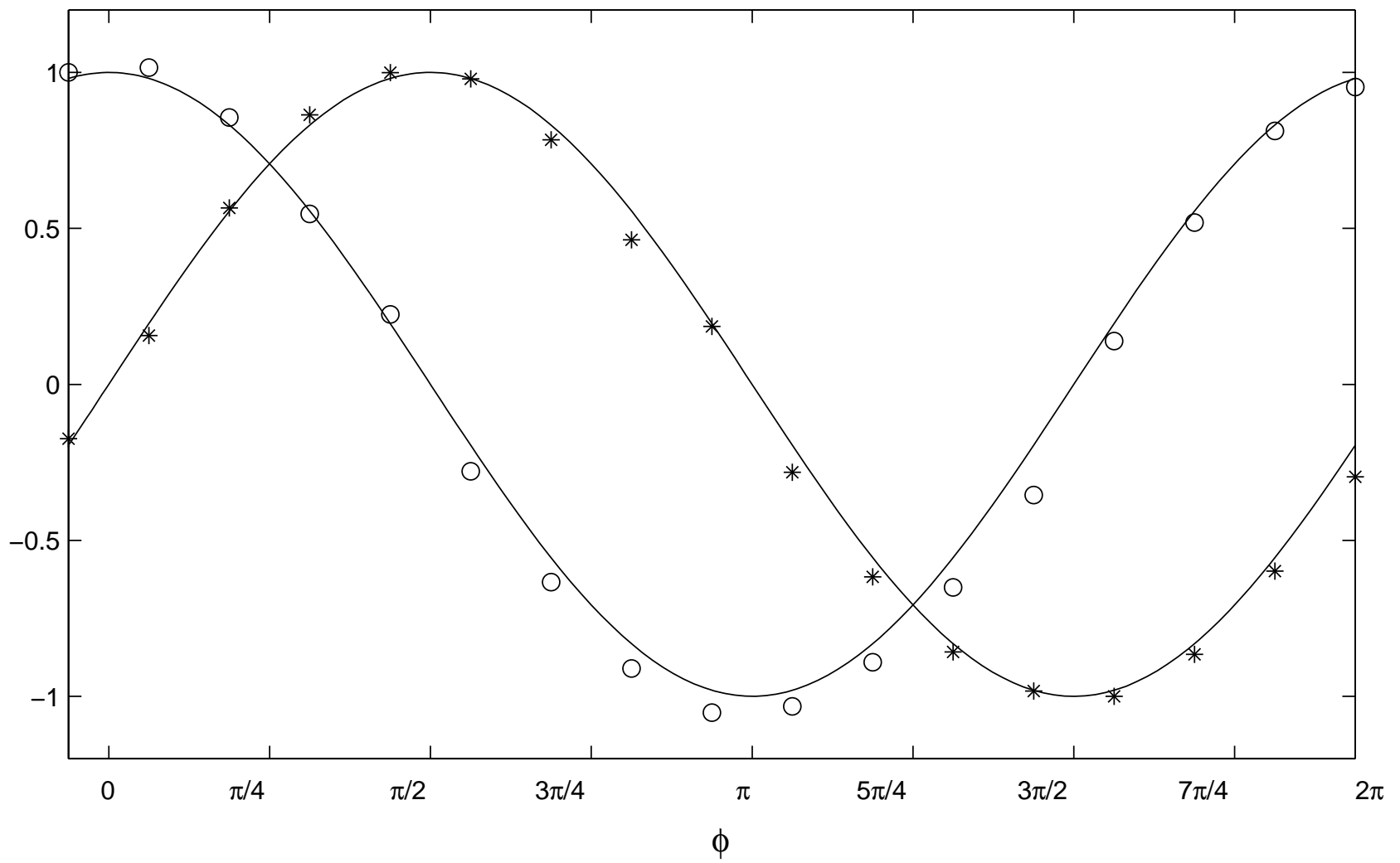

Fig. 2 